\title{
Analyze on GEO Satellites Output Power Variation
}

\author{
Jun ZHENG ${ }^{1}$ \\ ${ }^{1}$ Xi'an Satellite Control Center, Xi'an, Shaanxi, China \\ Email: ${ }^{1}$ zheng_raindrop@163.com
}

\author{
Hong CHEN ${ }^{2}$ \\ ${ }^{2}$ The Unit 63999 of Chinese People Liberation Army, Beijing, \\ China \\ Email: ${ }^{2}$ chehoner@163.com
}

\begin{abstract}
Solar arrays are the main source of energy used at present onboard satellites. Their energy resources largely determines the time of active life of on-orbit satellites. This paper sets up the output power variation model of solar arrays from the telemetry of three-axis stabilized GEO satellites made by China, and based on the model illustrates the influence of output power on on-orbit GEO satellites.
\end{abstract}

Keywords-Three-axis Stabilized GEO Satellites, Solar Arrays, Power; On-orbit Satellites

\section{Introduction}

The main source of energy of three-axis stabilized GEO satellites comes from solar arrays in active life time, so the output power variation of satellites affects the satellites application directly. Therefore, it is necessary to analyze the output power variation. However, it faces up some difficulties to establish a general power variation model. One reason is that some researchers study power variation with simulation only in laboratory ${ }^{[1]}$, and do not predict precisely power variation on-orbit satellites. Another reasons is solar arrays with different styles, different material made by different producers.

This paper aims at Chinese three-axis stabilized GEO satellites which are designed and manufactured by CAST. According with the telemetry from on-orbit CAST GEO satellites, we establish an output power variation model of solar arrays, and based on the model analyze the influence on on-orbit GEO satellites made by CAST.

\section{Short-term Output Power Variation of Solar Arrays}

There are $90 \%$ spacecrafts power are provided by electrical power sub-systems consisting of solar arrays and battery ${ }^{[2]}$.The solar arrays are key units of electrical power sub-system in GEO satellite. Solar arrays made by CAST are equipped by two equally sized foldable wings and each wing contains three rigid panels of passivated solar cells. The cells are arranged well according to several factors such as satellite power requirement, sun incidence angle and earth eclipse time. The cells absorb sun energy and transform it into electrical power.

Output power of solar arrays $P$ variates with solar radiation intensity $I$ and sun incidence angle $\theta_{s}$ :

$$
P=P_{\max } \cdot I \cdot \cos \theta_{s}
$$

Where $P_{\max }$ is maximum output power of solar arrays, $I$ is coefficient of solar radiation intensity and $\theta_{s}$ is sun incidence angle.

$\theta_{s}$ is cut angle between sun incidence vector $\vec{s}$ and sun panel normal vector $\vec{n}$ (Fig. 1). When $\theta_{s}$ reaches 0 , GEO satellite is in vernal equinox or in autumnal equinox and solar arrays generate maximum power; when $\theta_{s}$ reaches $23.44^{\circ}$, GEO satellite is in midwinter or in summer solstice and solar arrays generates minimum power (Fig. 2).
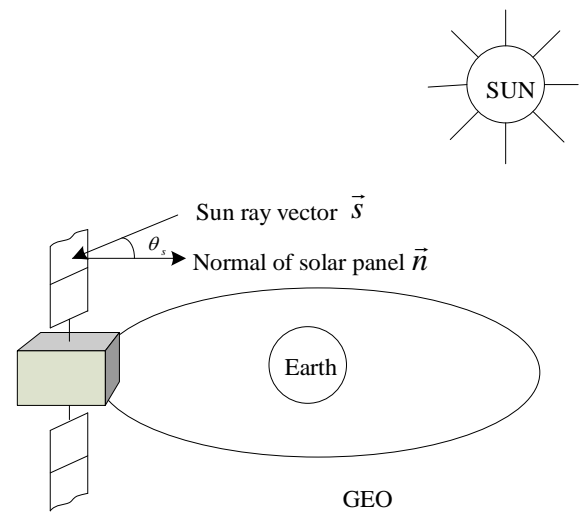

Three-axis stabilied GEO satellite

Fig.1 Solar arrays absorb sun energy and generate electrical power

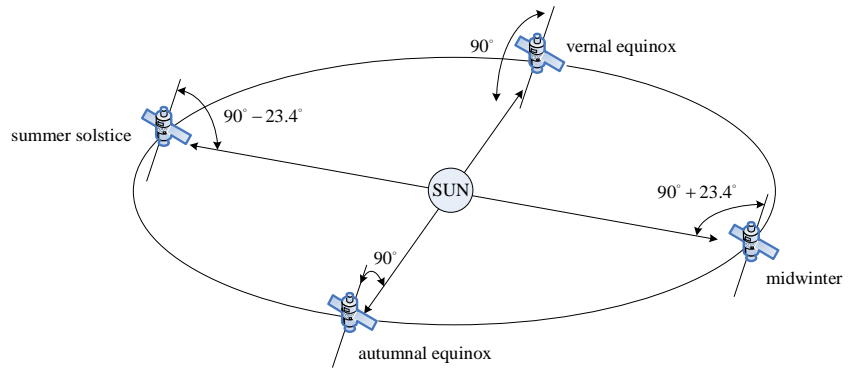

Fig.2 $\theta_{s}$ variation with different time

In Fig.1, Fig.2, the earth is running in ellipse orbit, so solar radiation intensity $I$ is periodic variation:

$$
I=1354+45 \cdot \cos \left(D \cdot \frac{360}{365.25}\right) \quad\left(\mathrm{W} / \mathrm{m}^{2}\right)
$$

Where $D$ is number of days from earth perihelion to current time. Every year the earth reaches perihelion approximately on Jan. 3rd, therefore, we transform $I$ into $J$ for normalization (Fig. 3): 


$$
J=\frac{I}{1353}
$$

Where $1353 \mathrm{~W} / \mathrm{m}^{2}$ is maximum solar radiation intensity on vernal (autumn) equinox

$$
\sin \left(\theta_{s}\right)=\sin 23.44 \cdot \sin \left[(D+101) \cdot \frac{360}{365.25}\right]
$$

As a result of earth rotation-axis slop relative to ecliptic plane normal, sun incidence angle $\theta_{s}$ oscillates for two times between $23.44^{\circ}$ and $-23.44^{\circ}$ every year (Fig.4).

If taking into account of solar radiation intensity variation and sun incidence angle variation merely, neglecting solar arrays attenuation coefficient, we can get the output power variation of solar arrays with time. (Fig. 5)

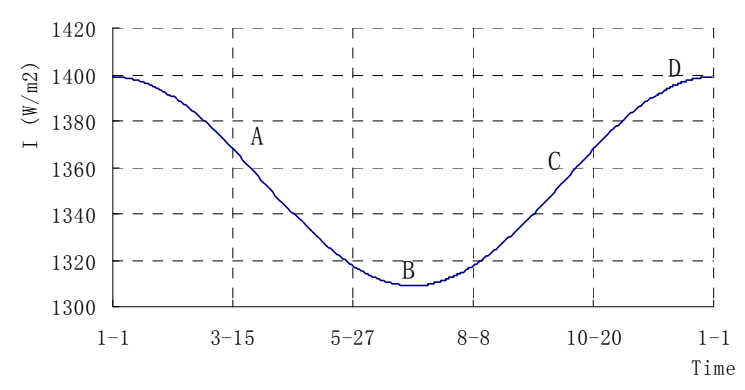

Fig.3 Solar radiation intensity variation in one year

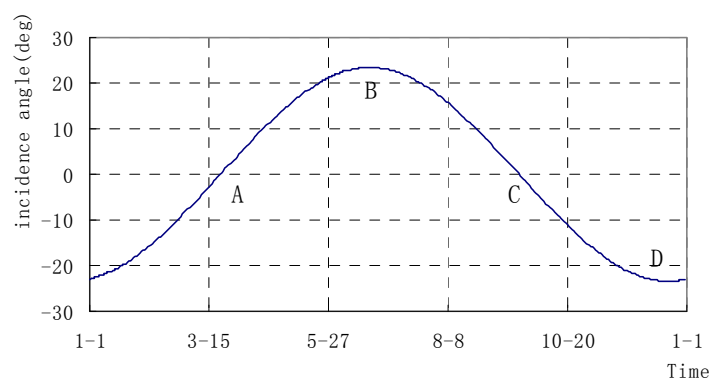

Fig.4 Incidence angle variation in one year

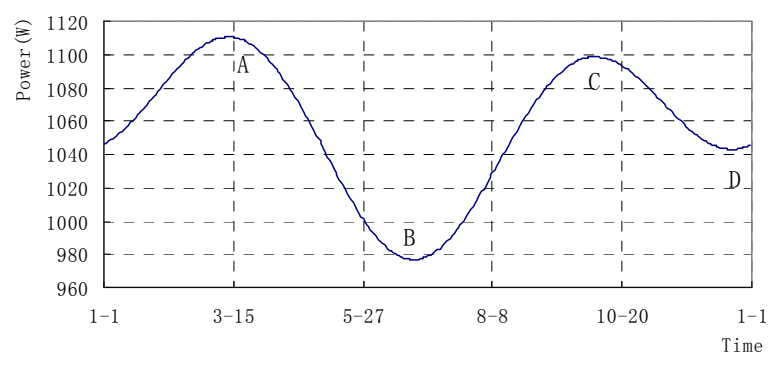

Fig.5 Output power variation in one year

Where in Fig.3, Fig.4, Fig.5, A is vernal equinox, B is summer solstice, $\mathrm{C}$ is autumnal equinox and $\mathrm{D}$ is midwinter.

\section{Long-term Output Power Variation of Solar Arrays}

Output power of solar arrays comes from the cells absorbing sun energy and transforming it into electrical power. Along with GEO satellite on-orbit continuously, the cells endure long-term damage coming from several reasons such as space ionizing radiation, solar ultraviolet emission, micro-meteorite impingement and so on. So the efficiency of light-electricity transformation is gradual decline and output power of solar arrays is decrease.

Table 1 is one CAST GEO satellite output power of solar arrays from telemetry on the same day in different year. The telemetry illustrates that output power of solar arrays is gradual decline clearly.

Table 1 One CAST GEO satellite actual output power from telemetry on the

\begin{tabular}{|c|c|}
\hline Time & Output power of solar arrays (W) \\
\hline $1997-6-11$ & 937.7252 \\
\hline 1998-6-11 & 861.7580 \\
\hline $1999-6-11$ & 801.3163 \\
\hline $2000-6-11$ & 780.2899 \\
\hline 2001-6-11 & 755.4629 \\
\hline 2002-6-11 & 742.4966 \\
\hline 2003-6-11 & 724.9405 \\
\hline
\end{tabular}
same day

Now we will analyze several factors of output power attenuation ${ }^{[3]}$ :

$$
P_{E O L}=P_{B O L} \cdot f_{\text {rad }} \cdot f_{u v} \cdot f_{\text {met }}
$$

Where $P_{E O L}$ is maximum output power of solar arrays in EOL (End Of Life), $P_{B O L}$ is maximum output power of solar arrays in BOL (Beginning Of Life), $f_{\text {rad }}$ is space ionizing radiation attenuation coefficient, $f_{u v}$ is solar ultraviolet emission attenuation coefficient, $f_{\text {met }}$ is micro-meteorite impingement attenuation coefficient.

$\eta=f_{\text {rad }} \cdot f_{u v} \cdot f_{\text {met }}$, where $\eta$ is general attenuation coefficient of solar arrays, we can prove ${ }^{[4]}$ that $\eta$ is exponential function:

$$
\eta=a+b \cdot e^{-\frac{D}{1000}}
$$

Where $D$ is number of days from earth perihelion to current time and rational number $\mathrm{a}$, b are coefficient.

We make use of the Least-Square-Algorithm to analyze the CAST GEO satellite telemetry on output power of solar arrays, and get the rational number coefficient a, b with MATLAB 6.5 ${ }^{[5]}$. So we can get the exponential function:

$$
\eta=0.696+0.304 \cdot e^{-\frac{D}{1000}}
$$

Therefore we acquire output power attenuation model (Eq. 8) of solar arrays from Eq. 1, Eq.2, Eq.3 and Eq. 7:

$$
P=P_{\max } \cdot J \cdot \eta \cdot \cos \theta_{s}
$$

Where $P_{\max }$ is maximum output power of solar arrays when GEO satellite on synchronous orbit, $J$ is normalized coefficient of solar radiation intensity; $\eta$ is general attenuation coefficient of solar arrays and $\theta_{s}$ is sun incidence angle. 


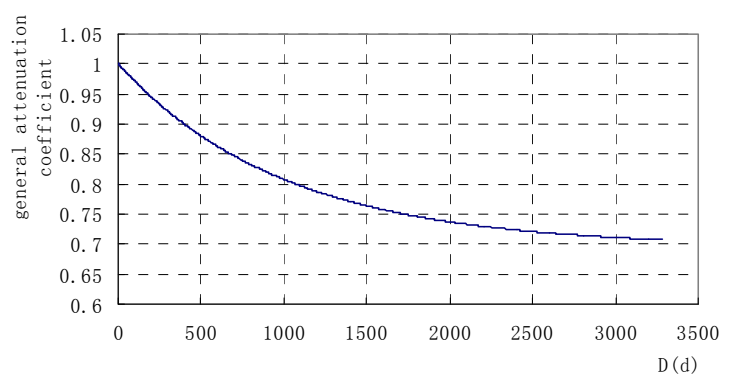

Fig.6 General attenuation coefficient of solar arrays variation

Now we make a simulation to predict the output power of solar arrays according to Eq.8.

Simulation condition:

1) simulation object: three-axis stabilized GEO satellite made by CAST;

2) $P_{\max }$ is given $1100 \mathrm{~W}$ (single wing maximum output power);

3) simulation time:2005-1-1 2013-12-31;

From the simulation result in Fig. 7, we know clearly that output power is decline continuously with time and we could predict the output power precisely for long time based on the model.

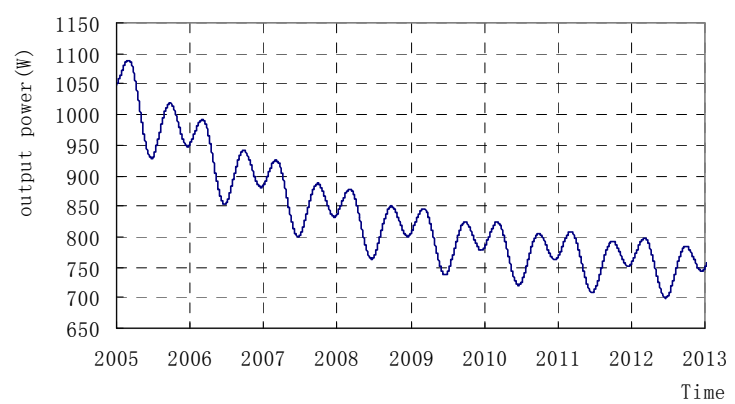

Fig.7 Output power of solar arrays variation with time

\section{Output Power of Solar Arrays Affects On-orbit} Satellites

When three-axis stabilized GEO satellite is in the stage of BOL, MOL (Middle Of Life), output power of solar arrays is able to meet the need of satellite load and keeps the satellite power balance ${ }^{[6]}$, although output power is gradual decrease. If the satellite is in EOL, its load does not change greatly relatively to in BOL and MOL. However, output power of solar arrays declines greatly, especially it is in summer solstice and midwinter, it is possible that the output power can not meet the load need and affect the satellite application. So we should pay great attention to satellite output power, predict the power wane time and take efficient scheme to avoid power wane.

In order to avoid power wane when on-orbit satellite is in EOL term, we set up a scheme (Fig. 8).

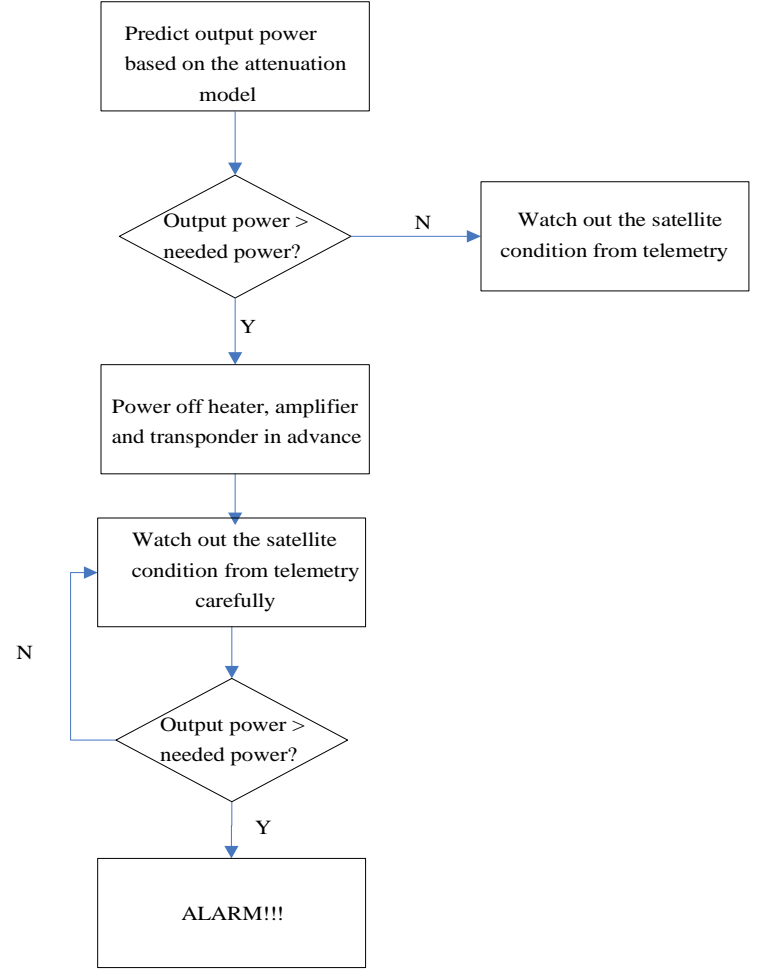

Fig. 8 Avoid power wane scheme

From Fig.8, we know that if output power can not meet the need according to output power prediction, we will shut down some facilities which consume too much power. However, if we often power off the heaters, amplifiers and transponders in satellite, it will reduce greatly the satellite applied value. So the engineers who design and manufacture satellites should take into account the power need and power supply, and guarantee the power supply and consumption balance.

\section{Conclusions}

Output power of solar arrays is key unit to three-axis stabilized GEO satellite. In this paper, we choose CAST GEO satellites as targets to analyze the short-term and long-term output power variation of solar arrays, and establish the output power attenuation model of solar arrays from CAST satellites telemetry. Based on the model, we set up the scheme to avoid power wane in satellites routine management.

\section{References}

[1] A. Shuvalov, G. S. Kochubei, V. V. Gubin, and N. A. Tokmak. Power Losses of Solar Arrays under the Action of an Environment in a Geosynchronous Orbit, Cosmic Research, Vol. 43, No. 4, 2005 pp. 259-267.

[2] XU Fu-xiang, Satellite Engineering, China Space Navigation Publishing Company, Beijing, China, Oct. 2002.

[3] MA Shi-jun, Satellite Electronic Power Technique, China Space Navigation Publishing Company, Beijing, China, Dec. 2001.

[4] Berlin Peter, The Geostationary Applications Satellite, Cambridge University Press, London, England, Jun.1988.

[5] CUI Yi, MATLAB5.3 Examples Elaborate, China Aviation Industry Publishing Company, Beijing, China, Jan. 2000.

[6] XIA Nan-yin, Space TT\&C System, National Defense Technology Publishing Company, Beijing, China, Oct. 2002. 